PROCEEDINGS OF THE

AMERICAN MATHEMATICAL SOCIETY

Volume 132, Number 2, Pages 417-428

S 0002-9939(03)07166-

Article electronically published on September 11, 2003

\title{
ON A THEOREM OF FAVARD
}

\author{
ZUOSHENG HU AND ANGELO B. MINGARELLI \\ (Communicated by Carmen C. Chicone)
}

\begin{abstract}
We obtain sufficient conditions for the existence of almost periodic solutions of almost periodic linear differential equations thereby extending Favard's classical theorem. These results are meant to complement previous results of the authors who have shown by means of a counterexample that the boundedness of all solutions is not, by itself, sufficient to guarantee the existence of an almost periodic solution to a linear almost periodic differential equation.
\end{abstract}

\section{INTRODUCTION}

This paper is motivated by a previous paper [7] in which the authors show that there exists a second order real linear differential equation on the line with almost periodic coefficients for which every solution is bounded but no nontrivial solution is almost periodic (in the classical sense of H. Bohr). This surprising phenomenon shows that boundedness, by itself, is not sufficient to guarantee the existence of almost periodic solutions of even the simplest linear equations. This is in sharp contrast with the periodic case in which Floquet's theory applies and gives the existence of almost periodic solutions in the case of bounded solutions. The authors' task of finding sufficient conditions for the existence of almost periodic solutions of linear systems of ordinary differential equations was undertaken by Jean Favard [4, a central figure in the theory of almost periodic differential equations whose work also inspired S. Bochner [1] in his extensions of the theory of almost periodic functions to Banach space. See [1, 5, 10] for further details. In order to guarantee the existence of at least one (always assumed nontrivial in the sequel) almost periodic solution for the system

$$
x^{\prime}=A(t) x+f(t),
$$

denoted by $\left(\mathrm{E}_{A, f}\right)$, Favard [4] established the following condition. If any nontrivial solution $x(t)$ of the system

$$
x^{\prime}=B(t) x
$$

satisfies

$$
\inf _{t \in R}|x(t)|>0,
$$

Received by the editors January 30, 2002 and, in revised form, June 20, 2002, September 11, 2002, and September 30, 2002.

2000 Mathematics Subject Classification. Primary 34A30, 34K14; Secondary 42A75.

Key words and phrases. Almost periodic functions, Favard's Theorem.

This research was partially supported by a research grant from NSERC Canada. 
for every $B(t)$ belonging to the uniform hull of the almost periodic matrix $A(t)$, where $f$ is also almost periodic and (1D) has a bounded solution, then there exists at least one solution of (1) which is almost periodic. There are results in various directions in the literature which imply Favard's Theorem (see [1, 5, 8, 10] and the references cited therein) but none of these results relax condition (31). A consequence of the results in this paper consists of the relaxation of condition (3) thereby producing an extension of Favard's Theorem under an assumption weaker than (3). R. A. Johnson [9] constructed an example that shows that if condition (3) does not hold, there does not exist any almost periodic solution of the system (1), but there may exist an almost automorphic solution. W. Shen and Y. Yi [10] established a general result on the existence of almost automorphic solutions for general linear systems. H. Ishii 8] and A. Haraux [6] considered nonlinear contractive almost periodic processes on a Banach space and, under some assumptions, they both established the existence of almost periodic solutions in different ways. In particular, Ishii [8] mentioned that when $A(t)$ is a skew symmetric $n \times n$ matrix for all $t \in R$, the corresponding result can imply Favard's Theorem. We will start within the framework of simple linear almost periodic differential equations because our main point is that we can weaken condition (3) by requiring instead that

$$
\inf _{t \in R}\left(\frac{1}{2 l} \int_{t-l}^{t+l}|x(s)| d s\right)>0
$$

where $l>0$ is some real number. On the other hand, for the first order equation, if we assume some uniformity, the weaker condition

$$
\inf _{t \in R}\left(\liminf _{l \rightarrow \infty} \frac{1}{2 l} \int_{t-l}^{t+l}|x(s)| d s\right)>0
$$

also guarantees the existence of an almost periodic solution of the corresponding differential equation. Our results can be extended to general almost periodic processes and these will appear in another paper.

\section{Existence of AN ALmost PERIOdic SOlution}

Throughout this paper, $R^{n}$ denotes an $n$-dimensional Euclidean space with the norm $|\bullet|$ and $C\left(R, R^{n}\right)$ denotes the continuous function space with the topology of uniform convergence. Following Bochner's definition 2 for an almost periodic function, we define the almost periodicity of a function as follows: A function $f \in C\left(R, R^{n}\right)$ is called (Bohr) almost periodic or, simply, almost periodic, if for any sequence $\left\{\sigma_{n}\right\}$ of $R$ there exists a subsequence $\left\{\sigma_{n}^{\prime}\right\} \subset\left\{\sigma_{n}\right\}$ such that the sequence $\left\{f\left(t+\alpha_{n}^{\prime}\right)\right\}$ converges to some function $g \in C\left(R, R^{n}\right)$ uniformly for $t \in R$. If $f, g \in C\left(R, R^{n}\right)$ and there exists a sequence $\left\{\alpha_{n}\right\} \subset R$ such that, for each $t \in R$,

$$
\lim _{n \rightarrow \infty} f\left(t+\alpha_{n}\right)=g(t),
$$

then we write this as $T_{\alpha_{n}} f=g$. If the limit (6) holds uniformly for all $t \in R$, we write $U T_{\alpha_{n}} f=g$. We use $H(f)$ to denote the uniform hull of $f$, i.e.,

$$
\mathcal{H}(f)=\left\{g \in C\left(R, R^{n}\right) \mid \text { there exists a sequence } \alpha_{n} \text { such that } U T_{\alpha_{n}} f=g\right\} .
$$

More generally, we say that the pair $(B, g) \in \mathcal{H}(A, f)$ if there exists a sequence $\alpha_{n}$ such that $U T_{\alpha_{n}} A=B$ and $U T_{\alpha_{n}} f=g$. For convenience, we label the equations 
$x^{\prime}=A(t) x+f(t)$ and $x^{\prime}=A(t) x$ by $\left(\mathrm{E}_{A, f}\right)$ and $\left(\mathrm{E}_{A}\right)$, respectively. The following preliminary results will be used in the sequel and are included for completeness.

Lemma 2.1 (S. Bochner [2]). Let $f \in C\left(R, R^{n}\right)$. Then $f$ is almost periodic on $R$ if and only if for any pair of sequences $\left\{\alpha_{n}\right\},\left\{\beta_{n}\right\} \subset R$, there exist two common subsequences $\left\{\alpha_{n}^{\prime}\right\} \subset\left\{\alpha_{n}\right\},\left\{\beta_{n}^{\prime}\right\} \subset\left\{\beta_{n}\right\}$ such that $T_{\alpha_{n}^{\prime}+\beta_{n}^{\prime}} f, T_{\alpha_{n}^{\prime}} f, T_{\alpha_{n}^{\prime}}\left(T_{\beta_{n}^{\prime}} f\right)$ all exist and $T_{\alpha_{n}^{\prime}+\beta_{n}^{\prime}} f=T_{\alpha_{n}^{\prime}}\left(T_{\beta_{n}^{\prime}} f\right)$.

Lemma 2.2 (A. M. Fink [5]). Let $f, g \in C\left(R, R^{n}\right)$. If $f$ is almost periodic on $R$ and $g \in \mathcal{H}(f)$, then $g$ is almost periodic on $R$.

Lemma 2.3 (A. M. Fink [5]). Let $x(t)$ be a bounded solution of $\left(\mathrm{E}_{A, f}\right)$. Then, for any $(B, g) \in \mathcal{H}(A, f)$, there is a sequence $\left\{\alpha_{n}\right\} \subset R$ and a solution $y(t)$ of the equation $\left(\mathrm{E}_{B, g}\right)$ such that $\lim _{n \rightarrow \infty} x\left(t+\alpha_{n}\right)=y(t)$ pointwise in $t \in R$.

Now for any $x(t) \in C\left(R, R^{n}\right)$ and $l>0$, we define

$$
L_{l}(x)=\sup _{t \in R}\left(\frac{1}{2 l} \int_{t-l}^{t+l}|x(s)|^{2} d s\right)^{\frac{1}{2}} .
$$

Lemma 2.4. If $u, v \in C\left(R, R^{n}\right)$ and there exists a sequence $\left\{\alpha_{n}\right\} \subset R$ such that $u\left(t+\alpha_{n}\right)$ converges to $v(t)$ for any $t \in R$, then for any $l>0, L_{l}(v) \leq L_{l}(u)$.

Proof. For any $l>0, t \in R, u\left(t+\alpha_{n}\right)$ converges to $v(t)$ uniformly on $[t-l, t+l]$. Therefore,

$$
\lim _{n \rightarrow \infty}\left(\frac{1}{2 l} \int_{t-l}^{t+l}\left|u\left(s+\alpha_{n}\right)-v(s)\right|^{2} d s\right)^{\frac{1}{2}}=0 .
$$

Using Minkowskii's inequality, we have

$$
\begin{aligned}
\left(\frac{1}{2 l}\right. & \left.\int_{t-l}^{t+l}|v(s)|^{2} d s\right)^{\frac{1}{2}} \\
& \leq\left(\frac{1}{2 l} \int_{t-l}^{t+l}\left|u\left(s+\alpha_{n}\right)\right|^{2} d s\right)^{\frac{1}{2}}+\left(\frac{1}{2 l} \int_{t-l}^{t+l}\left|u\left(s+\alpha_{n}\right)-v(s)\right|^{2} d s\right)^{\frac{1}{2}} \\
& \leq \sup _{t \in R}\left(\frac{1}{2 l} \int_{t-l}^{t+l}|u(s)|^{2} d s\right)^{\frac{1}{2}}+\left(\frac{1}{2 l} \int_{t-l}^{t+l}\left|u\left(s+\alpha_{n}\right)-v(s)\right|^{2} d s\right)^{\frac{1}{2}} .
\end{aligned}
$$

Letting $n \rightarrow \infty$, we have

$$
\left(\frac{1}{2 l} \int_{t-l}^{t+l}|v(s)|^{2} d s\right)^{\frac{1}{2}} \leq \sup _{t \in R}\left(\frac{1}{2 l} \int_{t-l}^{t+l}|u(s)|^{2} d s\right)^{\frac{1}{2}}=L_{l}(u),
$$

and thus $L_{l}(v) \leq L_{l}(u)$. This completes the proof of the lemma.

We assume that for any $(B, g) \in \mathcal{H}(A, f)$ and $(t, x) \in R \times R^{n}$, the equation $\left(\mathrm{E}_{B, g}\right)$ has a continuous solution through $(t, x)$ on $R$, and that there exists a $\left(t_{0}, x_{0}\right) \in R \times R^{n}$ such that the solution $x\left(t, t_{0}, x_{0}\right)$, denoted by $x_{0}(t)$, of the equation $\left(\mathrm{E}_{A, f}\right)$ is bounded on $R^{n}$. From these assumptions, Lemma 2.2 and Lemma 2.3, for any $(B, g) \in \mathcal{H}(A, f)$ there exists a sequence $\left\{\alpha_{n}\right\}$ and a solution $y(t)$ of the equation $\left(\mathrm{E}_{B, g}\right)$ such that $U T_{\alpha_{n}} A=B, U T_{\alpha_{n}} f=g$ and $T_{\alpha_{n}} x_{0}=y$ for $t \in R$. 
Now, by way of notation, we define $K=\operatorname{clco}\left\{x\left(t, t_{0}, x_{0}\right) \mid t \in R^{+}\right\}$where $\operatorname{clco}\{\cdots\}$ denotes the convex closure of the set $\{\cdots\}$, and

$$
\mathcal{F}_{B, g}=\left\{y(t) \mid y(t) \text { satisfies }\left(E_{B, g}\right) \text {, and } y(t) \in K \text {, for all } t \in R\right\} .
$$

By Lemma 2.3 for any $(B, g) \in \mathcal{H}(A, f), \mathcal{F}_{B, g}$ is not empty.

Lemma 2.5. For any $(B, g) \in \mathcal{H}(A, f)$ and any $l>0$, there exists $y_{0} \in \mathcal{F}_{B, g}$ such that

$$
L_{l}\left(y_{0}\right)=\inf _{u \in \mathcal{F}_{B, g}} L_{l}(u) \equiv \lambda .
$$

Proof. From Lemma 2.2 and Lemma $2.3 \mathcal{F}_{B, g}$ is sequentially compact in the topology of pointwise convergence. Therefore, $\mathcal{F}_{B, g}$ is closed under the topology of uniform convergence on compact subsets. We define

$$
\lambda_{n}=\inf _{u \in \mathcal{F}_{B, g}}\left(\sup _{|t| \leq n}\left(\frac{1}{2 l} \int_{t-l}^{t+l}|u(s)|^{2} d s\right)^{\frac{1}{2}}\right) .
$$

Then, $\lambda_{n} \leq \lambda$, and $\lim _{n \rightarrow \infty} \lambda_{n}=\lambda$. Take $u_{n} \in \mathcal{F}_{B, g}$ such that

$$
\sup _{|t| \leq n}\left(\frac{1}{2 l} \int_{t-l}^{t+l}\left|u_{n}(s)\right|^{2} d s\right)^{\frac{1}{2}} \leq \lambda_{n}+\frac{1}{n} .
$$

Therefore, there exists a $y_{0} \in \mathcal{F}_{B, g}$ such that $u_{n}(t)$ converges to $y_{0}(t)$ uniformly on any compact subset of $R$, i.e., $\left|u_{n}(t)-y_{0}(t)\right| \rightarrow 0$ uniformly on any compact subset of $R$. Now, for fixed $t \in R$, there is a positive integer $n>0$ such that $|t|<n$. An application of Minkowskii's inequality gives

$$
\begin{aligned}
& \left(\frac{1}{2 l} \int_{t-l}^{t+l}\left|y_{0}(s)\right|^{2} d s\right)^{\frac{1}{2}} \\
\leq & \left(\frac{1}{2 l} \int_{t-l}^{t+l}\left|y_{0}(s)-u_{n}(s)\right|^{2} d s\right)^{\frac{1}{2}}+\left(\frac{1}{2 l} \int_{t-l}^{t+l}\left|u_{n}(s)\right|^{2} d s\right)^{\frac{1}{2}} \\
\leq & \left(\frac{1}{2 l} \int_{t-l}^{t+l}\left|y_{0}(s)-u_{n}(s)\right|^{2} d s\right)^{\frac{1}{2}}+\sup _{|t| \leq n}\left(\frac{1}{2 l} \int_{t-l}^{t+l}\left|u_{n}(s)\right|^{2} d s\right)^{\frac{1}{2}} \\
\leq & \left(\frac{1}{2 l} \int_{t-l}^{t+l}\left|y_{0}(s)-u_{n}(s)\right|^{2} d s\right)^{\frac{1}{2}}+\lambda_{n}+\frac{1}{n} .
\end{aligned}
$$

Letting $n \rightarrow+\infty$, we see that the left-hand side of (8) is bounded above by $\lambda$, independently of $t \in R$, so that

$$
\sup _{t \in R}\left(\frac{1}{2 l} \int_{t-l}^{t+l}\left|y_{0}(s)\right|^{2} d s\right)^{\frac{1}{2}} \leq \lambda .
$$

Since $y_{0} \in \mathcal{F}_{B, g}$, from the definition of $\lambda$ we have that $L_{l}\left(y_{0}\right)=\lambda$. This completes the proof of the lemma.

Lemma 2.6. For any $(B, g),(D, h) \in \mathcal{H}(A, f)$ and any $l>0$, we have

$$
\inf _{u \in \mathcal{F}_{B, g}} L_{l}(u)=\inf _{u \in \mathcal{F}_{D, h}} L_{l}(u) \text {. }
$$


Proof. From Lemma [2.5. we can take $y_{B, g} \in \mathcal{F}_{B, g}$ and $y_{D, h} \in \mathcal{F}_{D, h}$ such that $L_{l}\left(y_{B, g}\right)=\inf _{u \in \mathcal{F}_{B, g}} L_{l}(u)$ and $L_{l}\left(y_{D, h}\right)=\inf _{u \in \mathcal{F}_{D, h}} L_{l}(u)$. We show that $L_{l}\left(y_{B, g}\right)=L_{l}\left(y_{D, h}\right)$. In fact, since $(B, g),(D, h) \in \mathcal{H}(A, f)$, we have $(D, h) \in$ $\mathcal{H}(B, g)$. From Lemma 2.3 there is a sequence $\left\{\alpha_{n}\right\}$ and a solution $\widetilde{y}_{D, h}(t)$ of the equation $\left(\mathrm{E}_{D, h}\right)$ such that $\widetilde{y}_{D, h}(t) \in \mathcal{F}_{D, h}$ and $y_{B, g}\left(t+\alpha_{n}\right)$ converges to $\widetilde{y}_{D, h}(t)$ pointwise in $R$. By Lemma 2.4, for any $l>0, L_{l}\left(\widetilde{y}_{D, h}\right) \leq L_{l}\left(y_{B, g}\right)$ and thus $L_{l}\left(y_{D, h}\right) \leq L_{l}\left(y_{B, g}\right)$. Similarly, we have $L_{l}\left(y_{B, g}\right) \leq L_{l}\left(y_{D, h}\right)$. This completes the proof of the lemma.

Lemma 2.7. Let $(B, g) \in \mathcal{H}(A, f)$ and let $l>0$ be some real number. If any nontrivial solution $y(t)$ of $\left(E_{B}\right)$ satisfies

$$
\left.\inf _{t \in R}\left(\frac{1}{2 l} \int_{t-l}^{t+l}|y(s)| d s\right)\right)>0,
$$

then there exists a unique solution $y_{B, g}(t)$ of the equation $\left(E_{B, g}\right)$ such that

$$
L_{l}\left(y_{B, g}\right)=\inf _{u \in \mathcal{F}_{B, g}} L_{l}(u) .
$$

Proof. Lemma 2.5 implies the existence of $y_{B, g}$. Now we show the uniqueness of $y_{B, g}$. Assuming the contrary, suppose that there exist two distinct trajectories $y_{B, g}$ and $z_{B, g}$ of the equation $\left(\mathrm{E}_{B, g}\right)$ satisfying (10). Let $w=\frac{1}{2}\left(y_{B, g}+z_{B, g}\right)$ and $v=\frac{1}{2}\left(y_{B, g}-z_{B, g}\right)$. Then, we have that $w$ is a solution of the equation $\left(\mathrm{E}_{B, g}\right)$, $w \in \mathcal{F}_{B, g}$, and that $v$ is a solution of the equation $\left(\mathrm{E}_{B}\right)$ and $v(t) \neq 0$ for all $t \in R$. We define

$$
\delta=\inf _{t \in R}\left(\frac{1}{2 l} \int_{t-l}^{t+l}|v(s)| d s\right),
$$

and $\lambda$ by the inf in Lemma 2.5.

Since $v$ is a nontrivial solution of $\left(\mathrm{E}_{B}\right)$, we must have $\delta>0$ by hypothesis (9). Furthermore, Cauchy's inequality gives

$$
\inf _{t \in R}\left(\frac{1}{2 l} \int_{t-l}^{t+l}|v(s)|^{2} d s\right)^{\frac{1}{2}} \geq \inf _{t \in R}\left(\frac{1}{2 l} \int_{t-l}^{t+l}|v(s)| d s\right),
$$

and so

$$
\inf _{t \in R}\left(\frac{1}{2 l} \int_{t-l}^{t+l}|v(s)|^{2} d s\right)^{\frac{1}{2}} \geq \delta>0
$$

Next,

$$
\begin{aligned}
& \left(\frac{1}{2 l} \int_{t-l}^{t+l}|w(s)|^{2} d s\right)+\left(\frac{1}{2 l} \int_{t-l}^{t+l}|v(s)|^{2} d s\right) \\
= & \left(\frac{1}{2 l} \int_{t-l}^{t+l} \frac{1}{2}\left|y_{B, g}(s)\right|^{2} d s\right)+\left(\frac{1}{2 l} \int_{t-l}^{t+l} \frac{1}{2}\left|z_{B, g}(s)\right|^{2} d s\right) \\
\leq & \sup _{t \in R}\left(\frac{1}{2 l} \int_{t-l}^{t+l} \frac{1}{2}\left|y_{B, g}(s)\right|^{2} d s\right)+\sup _{t \in R}\left(\frac{1}{2 l} \int_{t-l}^{t+l} \frac{1}{2}\left|z_{B, g}(s)\right|^{2} d s\right),
\end{aligned}
$$


from which we obtain

$$
\frac{1}{2 l} \int_{t-l}^{t+l}|w(s)|^{2} d s \leq \frac{1}{2} L_{l}^{2}\left(y_{B, g}\right)+\frac{1}{2} L_{l}^{2}\left(z_{B, g}\right)-\inf _{t \in R}\left(\frac{1}{2 l} \int_{t-l}^{t+l}|v(s)|^{2} d s\right) .
$$

Since $L_{l}\left(y_{B, g}\right)=L_{l}\left(z_{B, g}\right)=\lambda$, we have the following inequalities:

$$
\begin{aligned}
L_{l}^{2}(w) & \leq \frac{1}{2} L_{l}^{2}\left(y_{B, g}\right)+\frac{1}{2} L_{l}^{2}\left(z_{B, g}\right)-\inf _{t \in R}\left(\frac{1}{2 l} \int_{t-l}^{t+l}|v(s)|^{2} d s\right) \\
& \leq \frac{1}{2} \lambda^{2}+\frac{1}{2} \lambda^{2}-\delta^{2} \\
& <\lambda^{2}
\end{aligned}
$$

This contradicts the definition of $\lambda$ thereby completing the proof of the lemma.

Theorem 1. Let $A(t), f(t)$ be almost periodic on $R$. Suppose that there is some $l>0$ such that for every $(B, g) \in \mathcal{H}(A, f)$, any nontrivial solution $y(t)$ of the equation $\left(\mathrm{E}_{B}\right)$ satisfies (9). If there exists a $\left(t_{0}, x_{0}\right) \in R \times R^{n}$ such that $K=$ $\left\{x\left(t, t_{0}, x_{0}\right) \mid t \in R\right\}$ is bounded in $R^{n}$, then for any $(B, g) \in \mathcal{H}(A, f)$, there exists a solution of the equation $\left(\mathrm{E}_{B, g}\right)$ which is almost periodic on $R$.

Proof. From Lemmas 2.6 and 2.7, for any $(B, g) \in \mathcal{H}(A, f)$, there exists a unique solution $y_{B, g}(t)$ of the equation $\left(\mathrm{E}_{B, g}\right)$ such that $y_{B, g}(t) \in K$ for all $t \in R$ and

$$
L_{l}\left(y_{B, g}\right)=\inf _{u \in \mathcal{F}_{B, g}} L_{l}(u) .
$$

Now we show that $y_{B, g}(t)$ is almost periodic. From Bochner's theorem (Lemma 2.1) we have that for any pair of sequences $\left\{\alpha_{n}\right\},\left\{\beta_{n}\right\} \subset R$, there exist common subsequences $\left\{\alpha_{n}^{\prime}\right\}$ of $\left\{\alpha_{n}\right\}$ and $\left\{\beta_{n}^{\prime}\right\}$ of $\left\{\beta_{n}\right\}$ such that $B\left(t+\alpha_{n}^{\prime}\right)$ converges to some $D \in \mathcal{H}(A)$ and $g\left(t+\alpha_{n}^{\prime}\right)$ converges to some $h \in \mathcal{H}(f)$, and $B\left(t+\alpha_{n}^{\prime}+\beta_{n}^{\prime}\right)$ and $D\left(t+\beta_{n}^{\prime}\right)$ converge to some $E \in \mathcal{H}(A)$ and $g\left(t+\alpha_{n}^{\prime}+\beta_{n}^{\prime}\right)$ and $h\left(t+\beta_{n}^{\prime}\right)$ converge to some $e(t) \in \mathcal{H}(f)$. By Lemma 2.3. there exist a solution $y_{D, h}(t)$ of the equation $\left(\mathrm{E}_{D, h}\right)$, two solutions $y_{E, e}(t)$ and $z_{E, e}(t)$ of the equation $\left(\mathrm{E}_{E, e}\right)$ such that $y_{D, h} \in$ $\mathcal{F}_{D, h}, y_{E, e}, z_{E, e} \in \mathcal{F}_{E, e}$ and such that $y_{B, g}\left(t+\alpha_{n}^{\prime}\right)$ converges to $y_{D, h}(t), y_{D, h}\left(t+\beta_{n}^{\prime}\right)$ converges to $y_{E, e}(t)$, and $y_{B, g}\left(t+\alpha_{n}^{\prime}+\beta_{n}^{\prime}\right)$ converges to $z_{E, e}(t)$, respectively. From Lemma 2.4 we have

$$
L_{l}\left(y_{D, h}\right) \leq L_{l}\left(y_{B, g}\right), \quad L_{l}\left(y_{E, e}\right) \leq L_{l}\left(y_{D, h}\right), \quad \text { and } L_{l}\left(z_{E, e}\right) \leq L_{l}\left(y_{B, g}\right) .
$$

Lemmas 2.6] and 2.7 together imply that

$$
y_{E, e}(t)=z_{E, e}(t),
$$

for all $t \in R$. By Lemma 2.1, we have that $y_{B, g}(t)$ is almost periodic on $R$. This completes the proof of Theorem 1 .

\section{A SPECIAL CASE}

In this section, we discuss the simplest equation, the first order differential equation

$$
x^{\prime}=a(t) x+f(t),
$$

where $a(t)$ and $f(t)$ are scalar functions defined on $R$. We denote this equation by $\left(E_{a, f}\right)$. We always assume that $a(t)$ and $f(t)$ are almost periodic on $R$. First, using Theorem 1, we can specify the condition on $a(t)$ that will guarantee the existence of an almost periodic solution. 
Theorem 2. Assume that the equation $\left(\mathrm{E}_{a, f}\right)$ has a bounded solution. If there is an $l>0$ such that for any $b(t) \in \mathcal{H}(a)$ we have that

$$
\inf _{t \in R} \frac{1}{2 l} \int_{t-l}^{t+l} e^{\int_{0}^{s} b(u) d u} d s>0,
$$

then $\left(\mathrm{E}_{b, g}\right)$ has an almost periodic solution.

At the same time, if we assume some uniformity in the function $a(t)$, we can extend Favard's theorem under slightly weaker conditions than those presented in the previous section. To this end, we define

$$
L(u)=\sup _{t \in R}\left(\varlimsup_{l \rightarrow \infty}\left(\frac{1}{2 l} \int_{t-l}^{t+l}|u(s)|^{2} d s\right)^{\frac{1}{2}}\right)
$$

and establish an integral condition on the function $a(t)$ and $f(t)$.

Integral Condition. We say that $a$ and $f$ satisfy the Integral Condition if the following two conditions hold:

(1) For any $b \in \mathcal{H}(a)$, there is a real number $M>0$ such that, for all $t \in R$,

$$
\varlimsup_{l \rightarrow \infty}\left(\frac{1}{2 l} \int_{t-l}^{t+l} e^{2 \int_{0}^{s} b(\lambda) d \lambda} d s\right) \leq M .
$$

(2) Let $(b, g) \in \mathcal{H}(a, f)$ and $(d, h) \in \mathcal{H}(b, g)$. Let $u(t)$ and $v(t)$ be solutions of $\left(\mathrm{E}_{b, g}\right)$ and $\left(\mathrm{E}_{d, h}\right)$, respectively. If $u(t)$ is bounded on $R$ and, for some $\left\{\alpha_{n}\right\} \subset R, u\left(t+\alpha_{n}\right) \rightarrow v(t)$ pointwise for $t \in R$ as $n \rightarrow \infty$, then, for any $t \in R$,

$$
\lim _{n \rightarrow \infty}\left(\varlimsup_{l \rightarrow \infty}\left(\frac{1}{2 l} \int_{t-l}^{t+l}\left|u\left(s+\alpha_{n}\right)-v(s)\right|^{2} d s\right)^{\frac{1}{2}}\right)=0 .
$$

Lemma 3.1. Let $a$ and $f$ satisfy the Integral Condition, $(b, g) \in \mathcal{H}(a, f)$ and $\left\{v_{n}(t)\right\} \subseteq \mathcal{F}_{b, g}$. Then there is a subsequence, denoted by $\left\{v_{n}(t)\right\}$ again, and a solution $v(t)$ of $\left(\mathrm{E}_{b, g}\right)$ such that $v(t) \in \mathcal{F}_{b, g}$ and, for any $t \in R$,

$$
\lim _{n \rightarrow \infty}\left(\varlimsup_{l \rightarrow \infty}\left(\frac{1}{2 l} \int_{t-l}^{t+l}\left|v_{n}(s)-v(s)\right|^{2} d s\right)^{\frac{1}{2}}\right)=0 .
$$

Proof. Since $\mathcal{F}_{b, g}$ is sequentially compact, there is a function $v(t)$ and a subsequence of $\left\{v_{n}(t)\right\}$, denoted by $\left\{v_{n}(t)\right\}$ again, such that $v(t) \in \mathcal{F}_{b, g}$ and $v_{n}(t) \rightarrow v(t)$ pointwise as $n \rightarrow \infty$. Now,

$$
v_{n}(s)-v(s)=e^{\int_{0}^{s} b(u) d u}\left(v_{n}(0)-v(0)\right) .
$$

So, by (1) of the Integral Condition,

$$
\lim _{n \rightarrow \infty}\left(\varlimsup_{l \rightarrow \infty}\left(\frac{1}{2 l} \int_{t-l}^{t+l}\left|v_{n}(s)-v(s)\right|^{2} d s\right)^{\frac{1}{2}}\right)=0 \text { for any } t \in R .
$$

Lemma 3.2. Let $a, f$ satisfy the Integral Condition, $(b, g) \in \mathcal{H}(a, f)$. If $u(t)$ is a bounded solution of $\left(\mathrm{E}_{a, f}\right)$, then there exists a bounded solution $v(t)$ of $\left(\mathrm{E}_{b, g}\right)$ such that $L(v) \leq L(u)$. 
Proof. By Lemma 2.3 there exists a bounded solution $v(t)$ of $\left(\mathrm{E}_{b, g}\right)$ and a sequence $\left\{\alpha_{n}\right\}$ such that $a\left(t+\alpha_{n}\right) \rightarrow b(t), \quad f\left(t+\alpha_{n}\right) \rightarrow g(t)$ uniformly for $t \in R$ as $n \rightarrow \infty$ and $u\left(t+\alpha_{n}\right) \rightarrow v(t)$ pointwise for $t \in R$ as $n \rightarrow \infty$. From the Integral Condition, we have that for any $t \in R$,

$$
\lim _{n \rightarrow \infty}\left(\varlimsup_{l \rightarrow \infty} \frac{1}{2 l} \int_{t-l}^{t+l}\left|u\left(s+\alpha_{n}\right)-v(s)\right|^{2} d s\right)^{\frac{1}{2}}=0 .
$$

Using Minkowskii's inequality and suppressing some variables for ease of exposition, we have

$$
\begin{aligned}
\left(\frac{1}{2 l} \int_{t-l}^{t+l}|v(s)|^{2}\right)^{\frac{1}{2}} \leq & \left(\frac{1}{2 l} \int_{t-l}^{t+l}\left|u\left(s+\alpha_{n}\right)\right|^{2}\right)^{\frac{1}{2}} \\
& +\left(\frac{1}{2 l} \int_{t-l}^{t+l}\left|u\left(s+\alpha_{n}\right)-v(s)\right|^{2}\right)^{\frac{1}{2}}
\end{aligned}
$$

from which

$$
\begin{aligned}
& \left(\varlimsup_{l \rightarrow \infty} \frac{1}{2 l} \int_{t-l}^{t+l}|v(s)|^{2} d s\right)^{\frac{1}{2}} \\
\leq & \left(\varlimsup_{l \rightarrow \infty} \frac{1}{2 l} \int_{t-l}^{t+l}\left|u\left(s+\alpha_{n}\right)\right|^{2}\right)^{\frac{1}{2}}+\left(\varlimsup_{l \rightarrow \infty} \frac{1}{2 l} \int_{t-l}^{t+l}\left|u\left(s+\alpha_{n}\right)-v(s)\right|^{2}\right)^{\frac{1}{2}} \\
\leq & \sup _{t \in R}\left(\varlimsup_{l \rightarrow \infty} \frac{1}{2 l} \int_{t-l}^{t+l}|u(s)|^{2}\right)^{\frac{1}{2}}+\left(\varlimsup_{l \rightarrow \infty} \frac{1}{2 l} \int_{t-l}^{t+l}\left|u\left(s+\alpha_{n}\right)-v(s)\right|^{2}\right)^{\frac{1}{2}} .
\end{aligned}
$$

Letting $n \rightarrow \infty$, we get

$$
\left(\varlimsup_{l \rightarrow \infty} \frac{1}{2 l} \int_{t-l}^{t+l}|v(s)|^{2}\right)^{\frac{1}{2}} \leq \sup _{t \in R}\left(\varlimsup_{l \rightarrow \infty} \frac{1}{2 l} \int_{t-l}^{t+l}|u(s)|^{2}\right)^{\frac{1}{2}}=L(u),
$$

and thus $L(v) \leq L(u)$. This completes the proof of the lemma.

Lemma 3.3. Let $a, f$ satisfy the Integral Condition. For any $(b, g) \in \mathcal{H}(a, f)$, there exists $u_{0} \in \mathcal{F}_{b, g}$ such that $L\left(u_{0}\right)=\inf _{u \in \mathcal{F}_{b, g}} L(u)$.

Proof. Since $\mathcal{F}_{b, g}$ is sequentially compact in the topology of pointwise convergence, $\mathcal{F}_{b, g}$ is closed under the topology of uniform convergence on compact subsets. We define $\lambda$, as usual, by

$$
\lambda=\inf _{u \in \mathcal{F}_{b, g}} L(u)
$$

and

$$
\lambda_{n}=\inf _{u \in \mathcal{F}_{b, g}}\left(\sup _{|t| \leq n}\left(\limsup _{l \rightarrow \infty} \frac{1}{2 l} \int_{t-l}^{t+l}|u(s)|^{2} d s\right)^{\frac{1}{2}}\right) .
$$

Then, $\lambda_{n} \leq \lambda$ and $\lim _{n \rightarrow \infty} \lambda_{n}=\lambda$. Take $u_{n} \in \mathcal{F}_{b, g}$ such that

$$
\sup _{|t| \leq n}\left(\varlimsup_{l \rightarrow \infty} \frac{1}{2 l} \int_{t-l}^{t+l}\left|u_{n}(s)\right|^{2} d s\right)^{\frac{1}{2}} \leq \lambda_{n}+\frac{1}{n} \text {. }
$$


From Lemma 3.1, there exists a $u_{0} \in \mathcal{F}_{b, g}$ such that, for any $t \in R$,

$$
\lim _{n \rightarrow \infty}\left(\varlimsup_{l \rightarrow \infty}\left(\frac{1}{2 l} \int_{t-l}^{t+l}\left|u_{n}(s)-u_{0}(s)\right|^{2} d s\right)^{\frac{1}{2}}\right)=0 .
$$

Now, for fixed $t \in R$, there is a positive integer $n>0$ such that $|t|<n$. Minkowskii's inequality implies

$$
\begin{aligned}
& \left(\frac{1}{2 l} \int_{t-l}^{t+l}\left|u_{0}(s)\right|^{2} d s\right)^{\frac{1}{2}} \\
\leq & \left(\varlimsup_{l \rightarrow \infty} \frac{1}{2 l} \int_{t-l}^{t+l}\left|u_{0}(s)-u_{n}(s)\right|^{2} d s\right)^{\frac{1}{2}}+\left(\varlimsup_{l \rightarrow \infty} \frac{1}{2 l} \int_{t-l}^{t+l}\left|u_{n}(s)\right|^{2} d s\right)^{\frac{1}{2}} \\
\leq & \left(\varlimsup_{l \rightarrow \infty} \frac{1}{2 l} \int_{t-l}^{t+l}\left|u_{0}(s)-u_{n}(s)\right|^{2} d s\right)^{\frac{1}{2}}+\sup _{|t| \leq n}\left(\varlimsup_{l \rightarrow \infty} \frac{1}{2 l} \int_{t-l}^{t+l}\left|u_{n}(s)\right|^{2} d s\right)^{\frac{1}{2}} \\
(14) \leq & \left(\varlimsup_{l \rightarrow \infty} \frac{1}{2 l} \int_{t-l}^{t+l}\left|u_{0}(s)-u_{n}(s)\right|^{2} d s\right)^{\frac{1}{2}}+\lambda_{n}+\frac{1}{n} .
\end{aligned}
$$

Therefore,

$$
\left(\varlimsup_{l \rightarrow \infty} \frac{1}{2 l} \int_{t-l}^{t+l}\left|u_{0}(s)\right|^{2} d s\right)^{\frac{1}{2}} \leq\left(\varlimsup_{l \rightarrow \infty} \frac{1}{2 l} \int_{t-l}^{t+l}\left|u_{0}(s)-u_{n}(s)\right|^{2} d s\right)^{\frac{1}{2}}+\lambda_{n}+\frac{1}{n} .
$$

Letting $n \rightarrow+\infty$ we have, for any $t \in R$,

$$
\left(\varlimsup_{l \rightarrow \infty} \frac{1}{2 l} \int_{t-l}^{t+l}\left|u_{0}(s)\right|^{2} d s\right)^{\frac{1}{2}} \leq \lambda,
$$

so that

$$
\sup _{t \in R}\left(\varlimsup_{l \rightarrow \infty} \frac{1}{2 l} \int_{t-l}^{t+l}\left|u_{0}(s)\right|^{2} d s\right)^{\frac{1}{2}} \leq \lambda .
$$

From the definition of $\lambda$ and since $u_{0} \in \mathcal{F}_{b, g}$, we have that $L\left(u_{0}\right)=\lambda$. This completes the proof of the lemma.

Lemma 3.4. Let a, $f$ satisfy the Integral Condition. For any $(b, g),(d, h) \in \mathcal{H}$ $(a, f)$, we have

$$
\inf _{u \in \mathcal{F}_{b, g}} L(u)=\inf _{u \in \mathcal{F}_{d, h}} L(u) .
$$

Proof. From Lemma [3.3. we can take $y_{b, g} \in \mathcal{F}_{b, g}$ and $y_{d, h} \in \mathcal{F}_{d, h}$ such that $L\left(y_{b, g}\right)=\inf _{u \in \mathcal{F}_{b, g}} L(u)$ and $L\left(y_{d, h}\right)=\inf _{u \in \mathcal{F}_{d, h}} L(u)$. We show that $L\left(y_{b, g}\right)=$ $L\left(y_{d, h}\right)$. In fact, since $(b, g),(d, h) \in \mathcal{H}(a, f)$, we have $(d, h) \in \mathcal{H}(b, g)$. From Lemma 2.3 there is a sequence $\left\{\alpha_{n}\right\}$ and a solution $\widetilde{y}_{d, h}(t)$ of the equation $\left(\mathrm{E}_{d, h}\right)$ such that $\widetilde{y}_{d, h}(t) \in \mathcal{F}_{d, h}$ and $y_{b, g}\left(t+\alpha_{n}\right)$ converges to $\widetilde{y}_{d, h}(t)$ pointwise in $R$. By Lemma [3.2, $L\left(\widetilde{y}_{d, h}\right) \leq L\left(y_{b, g}\right)$ and thus $L\left(y_{d, h}\right) \leq L\left(y_{b, g}\right)$. Similarly, we have $L\left(y_{b, g}\right) \leq L\left(y_{d, h}\right)$. This completes the proof of the lemma.

Using the above three lemmas and an argument similar to that in Lemma 2.7 we can prove this final lemma. 
Lemma 3.5. Let $a, f$ satisfy the Integral Condition. If $(b, g) \in \mathcal{H}(a, f)$ are such that for any $t_{0} \in R$,

$$
\inf _{t \in R}\left(\liminf _{l \rightarrow \infty} \frac{1}{2 l} \int_{t-l}^{t+l} e^{\int_{t_{0}}^{t_{0}+s} b(\eta) d \eta} d s\right)>0,
$$

then there exists a unique solution $v_{b, g}$ of $\left(\mathrm{E}_{b, g}\right)$ such that $L\left(v_{b, g}\right)=\inf _{u \in \mathcal{F}_{b, g}} L(u)$.

For this special case, we can now formulate the following theorem in which Favard's condition is replaced by the weaker condition (15), where $t_{0} \in R$ is arbitrary but fixed.

Theorem 3. Let $a, f$ satisfy the Integral Condition. Suppose that for any $b \in \mathcal{H}(a)$, (15) holds. If $\left(\mathrm{E}_{a, f}\right)$ has a bounded solution, then for any $(b, g) \in \mathcal{H}(a, f),\left(\mathrm{E}_{b, g}\right)$ has an almost periodic solution on $R$.

The proof of this theorem is similar to that of Theorem 1 so we omit it.

\section{EXAMPLE}

Next, we construct a simple example in which Favard's condition (3) does not hold, but the key condition of Theorem 3 holds and the system has an almost periodic solution. Consider the first order linear differential equation $\left(\mathrm{E}_{a, f}\right)$, where $a(t), f(t): R \rightarrow R$ are almost periodic functions. Let $x\left(t, \tau, x_{0}\right)$ be the solution through $\left(\tau, x_{0}\right)$ of $\left(\mathrm{E}_{a, f}\right)$ for any $\tau, x_{0} \in R$. For any $b(t) \in \mathcal{H}(a)$ and $g(t) \in \mathcal{H}(f)$, there exists a solution $y\left(t, \tau, y_{0}\right)$ of the equation $\left(\mathrm{E}_{b, g}\right)$ through $\left(\tau, y_{0}\right)$. We assume that the function $a(t)$ satisfies the following three conditions:

(i) $a(t)$ is almost periodic on $R$,

(ii) $\int_{0}^{t} a(s) d s<0$ for all $t \in R$, and

(iii) there is a sequence $\left\{t_{n}\right\}$ such that $\lim _{n \rightarrow \infty} \int_{0}^{t_{n}} a(s) d s=-\infty$.

Consider the special differential equation $\left(\mathrm{E}_{a,-a}\right)$

$$
x^{\prime}=a(t)(x-1) .
$$

Obviously, this equation has an almost periodic solution $x(t) \equiv 1$, but the unperturbed equation, $x^{\prime}=a(t) x$, does not satisfy Favard's condition (3). However, we will show that this system satisfies the key condition of Theorem 3 for a special function $a(t)$. In order for the key condition of Theorem 3 to hold, we construct $a(t)$ as follows:

$$
\begin{gathered}
g_{1}(t)= \begin{cases}-\frac{1}{2} t, & t \in[0,2], \\
t-3, & t \in[2,3],\end{cases} \\
g_{2}(t)= \begin{cases}-\frac{1}{3^{2}-2^{1}} \frac{2}{2} t, & t \in\left[0,3^{2}-2^{1}\right], \\
-\frac{1}{2^{2-2}}, & t=8, \\
\text { linear, } & t \in\left[3^{2}-2^{1}, 3^{2}-2^{1}+1\right] \cup\left[3^{2}-1,3^{2}\right],\end{cases} \\
g_{n}(t)= \begin{cases}-\frac{1}{3^{n}-2^{n-1}} \times \frac{2}{n} t, & t \in\left[0,3^{n}-2^{n-1}\right], \\
-\frac{1}{2^{n-2},} & t \in\left[3^{n}-2^{n-1}+1,3^{n}-1\right], \\
\text { linear, } & t \in\left[3^{n}-2^{n-1}, 3^{n}-2^{n-1}+1\right] \cup\left[3^{n}-1,3^{n}\right] .\end{cases}
\end{gathered}
$$


Extend $g_{n}(t)$ to be odd and periodic with period $2 \times 3^{n}$. Then $g_{n}(t)$ satisfies the following conditions:

$$
\begin{aligned}
& \int_{0}^{t} g_{n}(s) d s \leq 0, \quad \text { for all } t \in R ; \\
& \qquad \sup _{t \in R}\left|g_{n}(t)\right|=\frac{n}{2^{n-2}} ; \quad \int_{0}^{t} g_{n}(s) d s \geq-\frac{1}{n},
\end{aligned}
$$

for all $t \notin\left[k \cdot 2 \cdot 3^{n}-2^{n-1}, k \cdot 2 \cdot 3^{n}+2^{n-1}\right], k=0, \pm 1, \pm 2, \ldots$, and

$$
\int_{0}^{3^{n}} g_{n}(s) d s \leq-1
$$

for each $n \in Z^{+}$. Letting $a_{n}(t)=\frac{1}{n} g_{n}(t)$, we see that $a_{n}(t)$ is a periodic function for each $n,\left|a_{n}(t)\right| \leq\left(n 2^{n-2}\right)^{-1}$, and setting $a(t)=\sum_{n=1}^{\infty} a_{n}(t)$, it follows that $a(t)$ is almost periodic on $R$. According to the definition of $a_{n}(t)$ and $a(t)$, we have that

$$
A(t)=\int_{0}^{t} a(s) d s=\sum_{n=1}^{\infty} \int_{0}^{t} a_{n}(s) d s \leq 0 .
$$

We let

$$
J=R \backslash \bigcup_{n=2}^{\infty} \bigcup_{k=-\infty}^{\infty}\left[3^{n}+k \cdot 2 \cdot 3^{n}-2^{n-1}, 3^{n}+k \cdot 2 \cdot 3^{n}+2^{n-1}\right] .
$$

Then, it is easy to show that for any $l>0$, the length of $J \cap[-l, l] \geq l$. Therefore, for any $t \in R$, we can take $l>0$ such that the length of $[t-l, t+l] \cap J \geq l$. Thus,

$$
\frac{1}{2 l} \int_{t-l}^{t+l} e^{A(s)} d s \geq \frac{1}{2 l} \int_{J \cap[t-l, t+l]} e^{A(s)} d s \geq \frac{1}{2} e^{-\delta}
$$

where $\delta=\sum_{n=1}^{\infty} n^{-2}$. Therefore, for any nontrivial solution $x(t)=x_{0} e^{A(t)}$ of $\left(\mathrm{E}_{a}\right)$, we have that

$$
\inf _{t \in R}\left(\liminf _{l \rightarrow \infty} \frac{1}{2 l} \int_{t-l}^{t+l}|x(s)| d s\right) \geq \frac{\left|x_{0}\right|}{2} e^{-\delta}>0 .
$$

It is easy to show that for any $b \in \mathcal{H}(a)$ the solution $y(t)$ of $\left(\mathrm{E}_{b}\right)$ also satisfies an inequality similar to (17).

Since $a$ is almost periodic and $\int_{0}^{t} a(s) d s \leq 0$, its mean value $m\{a\} \leq 0$. Thus, for $t_{0} \in R$, by the uniformity of this mean value, $c f$. [3], we obtain

$$
\lim _{t \rightarrow \infty} \frac{1}{t} \int_{t_{0}}^{t_{0}+t} a(s) d s \leq 0
$$

So, for any $b \in \mathcal{H}(a)$, we can show that $\int_{0}^{t} b(s) d s \leq 0$ and, therefore, for all $t \in R$,

$$
\varlimsup_{l \rightarrow \infty}\left(\frac{1}{2 l} \int_{t-l}^{t+l} e^{2 \int_{0}^{s} b(\lambda) d \lambda} d s\right) \leq 1 .
$$

Now, let $u(t)$ and $v(t)$ be bounded solutions of $\left(\mathrm{E}_{a,-a}\right)$, and $\left(\mathrm{E}_{b,-b}\right)$, respectively and let $\left\{\alpha_{n}\right\} \subset R$ such that $a\left(t+\alpha_{n}\right) \rightarrow b(t)$ uniformly and $u\left(t+\alpha_{n}\right) \rightarrow v(t)$ 
pointwise on $R$ as $n \rightarrow \infty$. Then

$$
\begin{aligned}
u\left(t+\alpha_{n}\right)-v(t)= & e^{\int_{\alpha_{n}}^{t+\alpha_{n}} a(s) d s}\left(u\left(\alpha_{n}\right)+\int_{\alpha_{n}}^{\alpha_{n}+t} e^{-\int_{\alpha_{n}}^{s} a(\lambda) d \lambda} a(s) d s\right) \\
& -e^{\int_{0}^{t} b(s) d s}\left(v(0)+\int_{0}^{t} e^{-\int_{0}^{s} a(\lambda) d \lambda} a(s) d s\right) \\
= & e^{\int_{0}^{t} a\left(s+\alpha_{n}\right) d s}\left(u\left(\alpha_{n}\right)+1\right)-e^{\int_{0}^{t} b(s) d s}(v(0)+1) .
\end{aligned}
$$

Hence, we can prove that, for any $t \in R$,

$$
\lim _{n \rightarrow \infty}\left(\varlimsup_{l \rightarrow \infty} \frac{1}{2 l} \int_{t-l}^{t+l}\left|u\left(s+\alpha_{n}\right)-v(s)\right|^{2} d s\right)^{\frac{1}{2}}=0 .
$$

Thus the pair $a,-a$ satisfies the Integral Condition.

\section{ACKNOWLEDGMENT}

The authors thank the referee for a critical review of this paper and for the changes suggested; in particular, the referee's elegant proof of Lemma 3.1.

\section{REFERENCES}

1. S. Bochner, Abstrakte fastperiodische funktionen, Acta Math., 61 (1933).

2. S. Bochner, A new approach to almost periodicity, Proc. Nat. Acad. Sci. U. S. A., 48 (1962), 2039-2043. MR 26:2816

3. H. Bohr, Almost Periodic Functions, Chelsea, New York, 1951, 114 pp. MR 8:512a

4. J. Favard, Leçons sur les fonctions presque-périodiques, Gauthier-Villars, Paris, 1933.

5. A. M. Fink, Almost Periodic Differential Equations, Lecture Notes in Mathematics, Vol. 377, Springer-Verlag, New York, 1974. MR 57:792

6. A. Haraux, Nonlinear Evolution Equations-Global Behavior of Solutions, Lecture Notes in Mathematics, Vol. 841, Springer-Verlag, New York, 1981. MR 83d:47066

7. Z. S. Hu and A. B. Mingarelli, On a question in the theory of almost periodic differential equations, Proc. Amer. Math. Soc., 127 (1999), 2665-2670. MR 99m:34095

8. H. Ishii, On the existence of almost periodic complete trajectories for contractive almost periodic processes, J. Differential Equations, 43 (1983), 66-72. MR 83c:34079

9. R. Johnson, Bounded solutions of scalar almost periodic linear equations, Illinois J. Math., 25 (1981), 632-643. MR 82k:34052

10. W. Shen and Y. Yi, Almost automorphic and almost periodic dynamics in skew-product semiflows, Part III, Applications to Differential Equations, Memoirs of the American Mathematical Society, 136, no. 647 (1998). MR 99d:34088

School of Mathematics and Statistics, Carleton University, Ottawa, Ontario, CANADA K1S 5B6

E-mail address: zhu@math.carleton.ca

School of Mathematics and Statistics, Carleton University, Ottawa, Ontario, CANADA K1S 5B6

E-mail address: amingare@math.carleton.ca 